\title{
Cardiovascular events in patients with mild autonomous cortisol secretion: analysis with artificial neural networks
}

\author{
Valentina Morelli',2, Serena Palmieri, ${ }^{1,2}$ Andrea Lania3, Alberto Tresoldi 1,3, \\ Sabrina Corbetta ${ }^{4}$, Elisa Cairoli ${ }^{1,2}$, Cristina Eller-Vainicher ${ }^{2}$, Maura Arosio ${ }^{1,2,5}$, \\ Massimiliano Copetti ${ }^{6}$, Enzo Grossi ${ }^{7}$ and lacopo Chiodini ${ }^{1}$
}

\begin{abstract}
${ }^{1}$ Department of Medical Sciences and Community Health, University of Milan, Milan, Italy, ${ }^{2}$ Unit of Endocrinology and Metabolic Diseases, IRCCS Cà Granda-Ospedale Maggiore Policlinico, Milan, Italy, ${ }^{3}$ Endocrine Unit, IRCCS Humanitas Clinical Institute, Humanitas University, Rozzano, Milan, Italy, ${ }^{4}$ Department of Biomedical Sciences, Unit of Endocrinology, University of Milan, IRCCS Istituto Ortopedico Galeazzi, Milan, Italy, ${ }^{5}$ Unit of Endocrine Diseases and Diabetology, Ospedale San Giuseppe, Gruppo Multimedica, Milan, Italy, ' Unit of Biostatistics ‘Casa Sollievo della Sofferenza', IRCCS, San Giovanni Rotondo, Foggia, Italy, and 'Semeion Center, Rome, Italy
\end{abstract}

Correspondence should be addressed to I Chiodini

Email

iacopo.chiodini@unimi.it

\begin{abstract}
Background: The independent role of mild autonomous cortisol secretion (ACS) in influencing the cardiovascular event (CVE) occurrence is a topic of interest. We investigated the role of mild ACS in the CVE occurrence in patients with adrenal incidentaloma (Al) by standard statistics and artificial neural networks (ANNs).

Methods: We analyzed a retrospective record of $518 \mathrm{Al}$ patients. Data regarding cortisol levels after $1 \mathrm{mg}$ dexamethasone suppression (1 mg DST) and the presence of obesity (OB), hypertension (AH), type-2 diabetes (T2DM), dyslipidemia (DL), familial CVE history, smoking habit and CVE were collected.

Results: The receiver-operating characteristic curve analysis suggested that $1 \mathrm{mg}$ DST, at a cut-off of $1.8 \mu \mathrm{g} / \mathrm{dL}$, had the best accuracy for detecting patients with increased CVE risk. In patients with $1 \mathrm{mg}-\mathrm{DST} \geq 1.8 \mu \mathrm{g} / \mathrm{dL}$ (DST+, $n=223$ ), age and prevalence of AH, T2DM, DL and CVE (66 years, 74.5, 25.9, 41.4 and 26.8\% respectively) were higher than that of patients with $1 \mathrm{mg}-\mathrm{DST} \leq 1.8 \mu \mathrm{g} / \mathrm{dL}$ (61.9 years, 60.7, 18.5, 32.9 and $10 \%$, respectively, $P<0.05$ for all). The CVE were associated with DST+ (OR: $2.46,95 \% \mathrm{Cl}: 1.5-4.1, P=0.01)$, regardless of T2DM, AH, DL, smoking habit, gender, observation period and age. The presence of at least two among AH, T2DM, DL and OB plus DST+ had 61.1\% sensitivity in detecting patients with CVE. By using the variables selected by ANNs (familial CVE history, age, T2DM, $\mathrm{AH}, \mathrm{DL}$ and DST+) $78.7 \%$ sensitivity was reached.

Conclusions: Cortisol after $1 \mathrm{mg}-\mathrm{DST}$ is independently associated with the CVE occurrence. The ANNs might help for assessing the CVE risk in Al patients.
\end{abstract}

\section{Introduction}

The condition of mild autonomous cortisol secretion (ACS) is characterized by an increased cortisol secretion in the absence of the classical signs of overt cortisol excess $(1,2)$, and it is found in up to $20 \%$ of patients bearing an incidentally discovered adrenal mass (adrenal incidentaloma, AI) $(3,4)$. In adults, mild ACS has a
두 2017 European Society of Endocrinology Printed in Great Britain
$0.2-2.0 \%$ prevalence and it seems to be associated with an increased risk of fragility fractures, dyslipidemia (DL), type 2 diabetes (T2DM), hypertension $(\mathrm{AH})$, cardiovascular events (CVE) and mortality $(5,6,7,8,9,10,11)$. However, since the improvement of the metabolic complications after the recovery from mild ACS has been not consistently 
reported $(12,13)$, no widely accepted guidelines are available on how to define mild ACS and to address the treatment in AI patients with subtle hypercortisolism (1, $15,16,17,18)$. Therefore, in the previous studies, the mild ACS condition was diagnosed on the basis of arbitrarily established criteria $(8,9,10)$ and, therefore, no data are available regarding the best test to be used for predicting the CVE occurrence in AI patients. The answer to this question could be given by assessing the association between the various parameters of cortisol secretion at different cut-offs with the CVE occurrence, regardless of an arbitrary previous diagnosis of mild ACS. However, this approach needs a large sample of AI patients to be studied.

Furthermore, in mild ACS the cardiovascular risk may be influenced by different comorbidities (i.e. DL, T2DM, $\mathrm{AH})$ and environmental factors interacting in nonlinear biological mechanisms. The understanding of the mild ACS role as a CVE risk factor is difficult even because most CVE risk factors may be caused by the mild ACS itself, and, at the same time, the incidence of both mild ACS and CVE increases with age (19). This kind of problem probably needs a specific mathematical approach, such as the artificial neural networks (ANNs), to be understood (20). As ANNs are artificial adaptive systems, able to modify their internal structure in relation to a function objective, they are suited for solving nonlinear problems. The ability to learn in an adaptive way (i.e. extracting from the data the information needed to gather a specific task) makes the ANNs a powerful tool for data analysis (21). In the past years, ANNs have been shown to improve the predictive value of standard statistics in many areas of medicine $(21,22,23,24,25,26,27,28,29)$. No studies have investigated the ability of ANNs in evaluating the factors influencing the CVE risk in patients with a low grade of cortisol excess. Therefore, the aim of the present study was to assess, in a large sample of AI patients, the best biochemical markers for detecting patients with increased CVE risk and to investigate the factors associated with the CVE events by using the ANNs and the standard statistical approach.

\section{Patients and methods}

\section{Patients}

In this observational multicenter study, we retrospectively analyzed the records of 1066 AI patients referred to the participating Endocrine Units between January 1996 and June 2016. Among these patients, 548 were not included due to the exclusion criteria. We excluded patients with bilateral adrenal masses, psychiatric diseases and alcoholism, or taking drugs influencing cortisol and dexamethasone metabolism or cortisol secretion, with signs or symptoms of overt cortisol excess (i.e. moon facies, striae rubrae, skin atrophy or buffalo hump), history of malignancy, infections, adrenal hemorrhage, pheochromocytoma, primary hyperaldosteronism and hyperparathyroidism, and infiltrative disease potentially affecting the adrenal glands. Eventually, 518 AI patients without signs of overt hypercortisolism were enrolled. Of all the patients enrolled in the present study, 95 subjects had been enrolled in a previous study reporting data of a long-term follow-up in AI (9).

All AI were discovered by CT scan, ultrasonography or MRI, performed for unrelated diseases. Ultrasound findings were confirmed with CT scan. All adrenal masses were $\geq 1 \mathrm{~cm}$ of diameter, and displayed a CT pattern consistent with benign adenoma (i.e. homogeneous texture, $<10$ Hounsfield Units, regular margins, size $<6 \mathrm{~cm}$ ).

In all patients, we measured 24-h urinary free cortisol (UFC), ACTH levels at 08:00 h, and serum cortisol levels at 08:00 h after $1 \mathrm{mg}$ overnight dexamethasone suppression test ( $1 \mathrm{mg}$-DST). The following parameters were recorded: age, gender, presence of obesity (OB), AH, T2DM, DL, familial CVE history and smoking habit. We recorded the CVEs occurrence (myocardial infarction, stroke, transient ischemic attack, angina pectoris, pulmonary embolism, intracerebral hemorrhage, peripheral artery disease) during the 10 years before the AI finding and during a variable follow-up period (mean: $161.8 \pm 45.1$, range: 120 426 months) after the AI finding. The observation period has been extended till 10 years before the AI finding since the diagnosis of overt hypercortisolism is done with 3-8 years of delay (30), which should be probably longer in scarcely symptomatic patients. The study complies with the Declaration of Helsinki and it has been approved by the Ethical Committee of Fondazione IRCCS Cà GrandaOspedale Maggiore Policlinico, Milan, Italy. An informed consent has been obtained from each patient.

\section{Methods}

In all patients, ACTH and serum and urinary cortisol levels were measured in each center using commercially available reagents. The intra- and inter-assay coefficients of variation were $<10 \%$ for all the assays. Increased UFC levels (h-UFC) were defined by levels above the upper limit of the normal values of each assay.

In the diabetic, dyslipidemic and hypertensive patients the CVEs, blood pressure and metabolic control 
were assessed by the reports of the cardiologists and diabetologists, who annually evaluated the patients. In the remaining patients, information was obtained by their general practitioner reports. Obesity was diagnosed in the presence of body mass index (BMI) above or equal to $30 \mathrm{~kg} / \mathrm{m}^{2}$ and T2DM by the WHO criteria (31). The patients with diabetes were considered well controlled in the presence of glycated hemoglobin below 7\% (32). Arterial hypertension was defined in the presence of systolic blood pressure $\geq 140 \mathrm{mmHg}$ and/or diastolic blood pressure $\geq 90 \mathrm{mmHg}$ and/or antihypertensive treatment (32). Blood pressure was considered well controlled in the presence of systolic and diastolic blood pressure below $130 \mathrm{mmHg}$ and $85 \mathrm{mmHg}$ respectively (33). Dyslipidemia was diagnosed in the presence of triglycerides levels $\geq 150 \mathrm{mg} / \mathrm{dL} \quad(1.7 \mathrm{mmol} / \mathrm{L})$, or high-density lipoprotein cholesterol levels $<40 \mathrm{mg} / \mathrm{dL}(1.0 \mathrm{mmoL} / \mathrm{L})$ in men and $<50 \mathrm{mg} / \mathrm{dL}(1.3 \mathrm{mmol} / \mathrm{L})$ in women or if any specific treatment was given (34). Dyslipidemia was considered well controlled in the presence of low density lipoprotein (LDL) below 130, 100 and $70 \mathrm{mg} / \mathrm{dL}$ in patients without T2DM, with T2DM and with T2DM plus cardiovascular complications respectively. In each patient a comorbidities score was calculated by adding up the comorbidities (T2DM, AH, DL and OB) altogether. Current smokers were individuals who smoked any tobacco (including beedies, pipes and other forms) during the observation period (35). The participating Endocrine Units were asked to provide each patient's data regarding the presence or absence of glycemia, LDL and blood pressure at target.

\section{Statistical analysis}

\section{Standard statistical analyses}

Statistical analyses were performed using SPSS version 21.0 package (SPSS). Descriptive statistics were reported as mean \pm standard deviation (s.D.), unless differently specified, or frequency and percentages. Categorical variables were compared by $\chi^{2}$ test. Continuous variables were compared among groups using one-way ANOVA. Multiple comparisons were addressed using the Bonferroni approach. The bivariate associations were assessed using the Spearman or Pearson Correlation as appropriate. The receiver-operating characteristic (ROC) curve analysis was used to assess the optimal cut-off of the cortisol levels after $1 \mathrm{mg}$-DST or of the comorbidity score providing the best diagnostic accuracy (optimizing jointly sensitivity, SN and specificity, SP) for detecting patients with increased CVE risk.
Multivariable logistic regression analyses were performed to test the independent association between the CVE occurrence and the cortisol levels after $1 \mathrm{mg}$-DST above or equal to the cut-off obtained by ROC curve, adjusting for age, gender, familial CVE history, duration of observation, smoking habit and the presence of T2DM, $\mathrm{AH}$ and DL separately taken or as a comorbidity score equal to or above the cut-off obtained by the ROC curve. Risk was reported as odds ratios (ORs) along with their 95\% confidence intervals (95\% CIs).

Potential nonlinear association between $1 \mathrm{mg}$ DST and CVE events was tested using the Box-Tidwell approach (36) (by creating an interaction term between the continuous variable and its own natural log and adding the interaction term to the model). In addition, we compared linear and quadratic models in terms of Akaike's information criterion. $P$ values of $<0.05$ were considered as statistically significant.

\section{Unsupervised ANNs: the Auto-Contractive Map}

Complex mathematical networks can help us in establishing the hierarchy of variables within a specific set. We adopted the Auto-Contractive Map system (Auto-CM), that is a fourth generation unsupervised ANN able to highlight the links among variables with a graph based on the minimum spanning tree (MST) theory, where distances among variables reflect the weights of the ANN (20). The Auto-CM system finds, by a specific learning algorithm, a square matrix of 'similarities' (weights mathematically speaking) among the variables of the dataset (21). Once the Auto-CM weights matrix is obtained, it is then filtered by MST, which shows, among the huge number of possible ways to connect the variables in a tree, the shortest possible combination. In the MST, every link able to generate a cycle into the graph is eliminated, irrespective of its strength of association, and this results in a simplified graph. The assumption is that, since all biological systems tend naturally to the minimal energetic states, this graph expresses the fundamental biological information of the system. The ultimate goal of this data mining model is to discover hidden trends and associations among variables, since this algorithm is able to create a semantic connectivity map in which nonlinear associations are preserved and explicit connection schemes are described. This approach gives the map of the relevant connections between and among variables and the principal hubs of the system. 
The learning algorithm of Auto-CM may be summarized in four orderly steps: (a) signal transfer from the input into the hidden layer; (b) adaptation of the connections value between the input layer and the hidden layer; (c) signal transfer from the hidden layer into the output layer; (d) adaptation of the connections value between the hidden layer and the output layer. The Auto-CM does not have initial weights posed at random and they start always by the same value. Therefore, the resulting graph is reproducible along many possible runs $(20,21)$. In simple words Auto-CM 'spatialises' the correlation among different variables ('closeness'), and converts it into a compelling graph that identifies only the relevant associations and organizes them into a coherent picture, building a complex global picture of the whole pattern of variation.

We choose arbitrarily to consider as relevant hubs those variables that showed at least five connections with other variables in the network. Moreover, we defined a 'central node' the inner node that is the last remaining after bottom-up recursively pruning away the 'leaves' nodes (i.e. the isolated ends of the graph) $(20,21,22)$.

\section{Supervised ANNs}

To evaluate the possibility to predict outcome (CVE yes vs CVE not) starting from risk factors on study we used supervised artificial neural networks developed in Semeion Research Center using the training and testing validation protocol.

Training and testing validation protocol implies the following steps:

- Subdivision of the data set into two sub-samples: subset A and subset B. In the first run, subset A is used as the Training Set and the subset B as the Testing Set.

- Application of ANN on the Training Set. In this phase, the ANN learns to associate the input variables with those indicated as targets.

- At the end of the training phase, the weights matrix produced by the algorithm is saved and frozen together with all of the other parameters used for the training.

- The Testing Set, is then shown to a virgin twin (same architecture and base parameters) ANN with the same weights matrix of the trained ANN, acting as final classifier, so that for each case of the testing set, new for the ANN, the classifier expresses a classification in one of the two targets based on the learning previously carried out during training. This operation takes place for all records in testing each and result (right or wrong classification) is not communicated to the classifier.
This allows to assess the generalization ability of trained ANN.

- In a second run another virgin $\mathrm{ANN}$ is applied to subset $\mathrm{B}$, which is used as training subset and then to subset $\mathrm{A}$ which is used as a testing subset.

- Therefore, the results are relevant to two sequences of training-testing protocol: $\mathrm{A}-\mathrm{B}$ and $\mathrm{B}-\mathrm{A}$. Results are expressed in terms of sensitivity (correct classification of CVE presence), specificity (correct classification of CVE absence), global accuracy (arithmetic mean between sensitivity and specificity). Overall results are expressed as average of the two experiments.

This crossover procedure allows to classify blindly all the records with the trained algorithm ensuring the generalization capability of the model on records never seen before.

The split of original data set in two subsets has not been obtained with a simple random split but with an evolutionary algorithms called TWIST (Training With Input Selection and Testing), recently described (23) and developed in a special non-commercial research software at the Semeion Research Center in Rome, Italy.

\section{TWIST algorithm}

TWIST algorithm is a complex evolutionary algorithm able to look for the best distribution of the global dataset divided in two optimally balanced subsets containing a minimum number of input features useful for optimal pattern recognition. TWIST is an evolutionary algorithm based on a seminal paper about Genetic Doping Systems, already applied to medical data with very promising results $(23,24)$. Usually TWIST evolutionary system is constituted by a population of Multilayer Perceptrons. Each ANN has to learn a subset of the global dataset and has to be tested in a blind way with another subset. TWIST selected some of the original attributes and generated two optimal subsets for training and testing with a reduced number of variables. We then applied the training-testing protocol to the reduced dataset to verify the predictive capacity of supervised back propagation neural network on the attributes selected by TWIST.

\section{Results}

\section{Classical statistical analysis}

We found the occurrence of myocardial infarction, stroke, transient ischemic attack, angina pectoris, pulmonary embolism, intracerebral hemorrhage and 
peripheral artery disease in the $38.9,15.6,13.3,7.8,3.3$, 1.1 and $20 \%$ of patients respectively. Table 1 reports the clinical and biochemical characteristics of patients with and without CVE (CVE+ and CVE- respectively). The adenoma size, duration of observation period, number of interval visits and prevalence of postmenopausal females and of subjects with positive familial CVE history were comparable between the two groups. As expected, CVE+ patients were elder and more frequently males, smokers, hypertensive, diabetic and dyslipidemic than CVE- patients. However, BMI, the prevalence of $\mathrm{OB}$ and of uncontrolled glycemia, blood pressure and LDL levels were comparable between the two groups. The cortisol levels after $1 \mathrm{mg}$-DST were higher in CVE+ than those in CVE- patients, whereas the ACTH levels and the prevalence of h-UFC levels were comparable between the two groups. The logistic regression analysis showed that the CVE occurrence was associated with the cortisol levels after $1 \mathrm{mg}$-DST taken as a continuous variable, regardless of age, gender, duration of observation, familial CVE history, smoking habit, T2DM, AH and DL (Table 2). Subdividing the whole sample of patients on the basis of $1 \mathrm{mg}$-DST above $1.8 \mu \mathrm{g} / \mathrm{dL}$ (the widely used cut-off for excluding hypercortisolism, $n=316$ ), between 1.8 and $5 \mu \mathrm{g} / \mathrm{dL}$ (the cut-offs considered by most authors as suggestive for possible hypercortisolism, $n=115$ ) and above $5 \mu \mathrm{g} / \mathrm{dL}$ (the cut-off for diagnosing hypercortisolism, $n=87$ ), we found that the CVE prevalence progressively increased from the first to the third group of patients $(10.8,21.7$, $35.6 \%$ respectively, $P$ for trend $<0.0001)$. Furthermore, subdividing the whole sample on the basis of quartiles of $1 \mathrm{mg}$ DST (I quartile: $<1.15 \mu \mathrm{g} / \mathrm{dL}, n=129$; II quartile: $1.16-1.5 \mu \mathrm{g} / \mathrm{dL}, \quad n=128 ; \quad$ III quartile: $1.6-2.4 \mu \mathrm{g} / \mathrm{dL}$, $n=133$; IV quartile: $>2.4 \mu \mathrm{g} / \mathrm{dL}, n=128$ ), we found that the CVE prevalence was similar between the I and II quartiles $(9.3 \%$ and $9.4 \%$ respectively) and between the III and IV quartiles ( $21.8 \%$ and $28.9 \%$ respectively) whereas it was significantly higher in the III and IV quartiles as compared with the I and II quartiles, $P=0.01$ for both comparisons (Fig. 1).

We also subdivided only the sample of patients with $1 \mathrm{mg}$-DST above $1.8 \mu \mathrm{g} / \mathrm{dL}$ in tertiles on the basis of the cortisol levels after $1 \mathrm{mg}$ DST (I tertile: $1.81-2.2 \mu \mathrm{g} / \mathrm{dL}$, $n=72$; II tertile: $2.21-3.1 \mu \mathrm{g} / \mathrm{dL}, n=73$; III tertile: $>3.1 \mu \mathrm{g} /$ $\mathrm{dL}, n=75)$. The CVE prevalence tended to be higher in the III tertile (34.7\%) as compared with I $(23.6 \%, P=0.09)$ and II $(21.9 \%, P=0.08)$ ones, although the statistical difference was not reached.

Finally, the Box-Tidwell test refused the hypothesis of a nonlinear association between CVS events and 1 mg DST $(P=0.73)$. Akaike's information criterion values for linear and quadratic models were 402.01 and 403.76 respectively, thus confirming the linear hypothesis.

Table 1 Clinical and biochemical parameters of patients with and without CVE. Data are mean \pm s.d. with range in parenthesis or absolute number with percentage in parenthesis.

\begin{tabular}{|c|c|c|c|}
\hline & CVE+ group $(n=90) n=231$ & CVE- group $(n=428)$ & $\boldsymbol{P}$ \\
\hline Age (years) & $68.3 \pm 9.8(20-84)$ & $62.7 \pm 10.4(33-86)$ & $<0.001$ \\
\hline Male/female gender & $47 / 43(52 / 48)$ & $157 / 271(37 / 63)$ & 0.009 \\
\hline Postmenopausal females & $43(47.8)$ & $229(53.5)$ & 0.354 \\
\hline BMI $\left(\mathrm{kg} / \mathrm{m}^{2}\right)$ & $28.1 \pm 4.5(20-40)$ & $27.5 \pm 4.8(18-40)$ & 0.279 \\
\hline Diameter of adenoma (mm) & $26 \pm 9.7(10-55)$ & $24.4 \pm 9.5(10-60)$ & 0.164 \\
\hline Duration of observation period (months) & $164.7 \pm 49.3(121-353)$ & $161.2 \pm 44.2(120-426)$ & 0.425 \\
\hline Visits during the observation period & $13.7 \pm 4.1(10-29)$ & $13.4 \pm 3.7(10-36)$ & 0.524 \\
\hline Patients with familial CVE history & $27(30)$ & $123(28.7)$ & 0.799 \\
\hline Smokers & $47(52)$ & $173(40)$ & 0.027 \\
\hline ACTH $(p g / m L)$ & $14.3 \pm 9(1.6-55)$ & $14.8 \pm 10.4(1-55)$ & 0.696 \\
\hline $1 \mathrm{mg}-\mathrm{DST}(\mu \mathrm{g} / \mathrm{dL})$ & $2.5 \pm 1.4(0.5-7.2)$ & $1.9 \pm 1.4(0.1-11.5)$ & $<0.001$ \\
\hline h-UFC patients & $6(6.7)$ & $36(8.4)$ & 0.676 \\
\hline Hypertensive patients & $83(92)$ & $262(61)$ & $<0.001$ \\
\hline Blood pressure not at target ${ }^{a}$ & $41(46.5)$ & $178(41.5)$ & 0.557 \\
\hline Diabetic patients & $39(43)$ & $73(17)$ & $<0.001$ \\
\hline Glycemia not at target ${ }^{b}$ & $9(10)$ & $25(5.8)$ & 0.160 \\
\hline Dyslipidemic patients & $51(57)$ & $138(32)$ & $<0.001$ \\
\hline LDL not at target & $25(27.8)$ & $91(21.3)$ & 0.210 \\
\hline Obese subjects & $27(30)$ & $141(33)$ & 0.622 \\
\hline
\end{tabular}

${ }^{a}$ Among hypertensive patients; bamong diabetic patients.

$1 \mathrm{mg}$-DST, serum cortisol levels after $1 \mathrm{mg}$ dexamethasone suppression test; ACTH, adrenocorticotroph hormone; BMI, body mass index; CVE, cardiovascular event; h-UFC, urinary free cortisol levels above normal reference range; LDL, low density lipoprotein; SI conversion factors, cortisol $\times 27.59$, АСТH $\times 0.22$; smokers, individuals who smoked any tobacco (including beedies, pipes and other forms) during the observational period. 
Table 2 Association between serum cortisol levels after $1 \mathrm{mg}$ dexamethasone suppression test and the occurrence of cardiovascular event after adjustment for the presence of comorbidities and of other possible contributing factors using the multivariate logistic regression analysis.

\begin{tabular}{|c|c|c|c|}
\hline & $\overline{\text { Odds ratio }}$ & $95 \% \mathrm{Cl}$ & $P$ value \\
\hline Female gender & 1.6 & $0.9-2.7$ & 0.09 \\
\hline Age (1 year increase) & 1.04 & $1.0-1.1$ & 0.01 \\
\hline $\begin{array}{l}\text { Familial CVE history (presence vs } \\
\text { absence) }\end{array}$ & 0.94 & $0.6-1.9$ & 0.81 \\
\hline T2DM (presence vs absence) & 1.9 & $1.1-3.4$ & 0.02 \\
\hline AH (presence vs absence) & 4.3 & $1.9-9.9$ & 0.001 \\
\hline DL (presence vs absence) & 1.8 & $1.1-3.1$ & 0.03 \\
\hline Smoke (presence vs absence) & 1.9 & $1.1-3.2$ & 0.03 \\
\hline $\begin{array}{l}\text { Duration of observation period } \\
\text { (1 month increase) }\end{array}$ & 1.0 & $1.0-1.0$ & 0.844 \\
\hline $1 \mathrm{mg}-\mathrm{DST}(1 \mu \mathrm{g} / \mathrm{dL}$ increase $)$ & 1.3 & $1.1-1.5$ & 0.004 \\
\hline
\end{tabular}

$1 \mathrm{mg}$-DST, serum cortisol levels at 08:00 $\mathrm{h}$ after $1 \mathrm{mg}$ dexamethasone suppression test; $\mathrm{AH}$, arterial hypertension; comorbidities score, sum of the comorbidities (type 2 diabetes, arterial hypertension, dyslipidemia; $\mathrm{DL}$, dyslipidemia; smoke, smoke of any tobacco (including beedies, pipes and other forms) during the observational period; T2DM, type 2 diabetes mellitus.

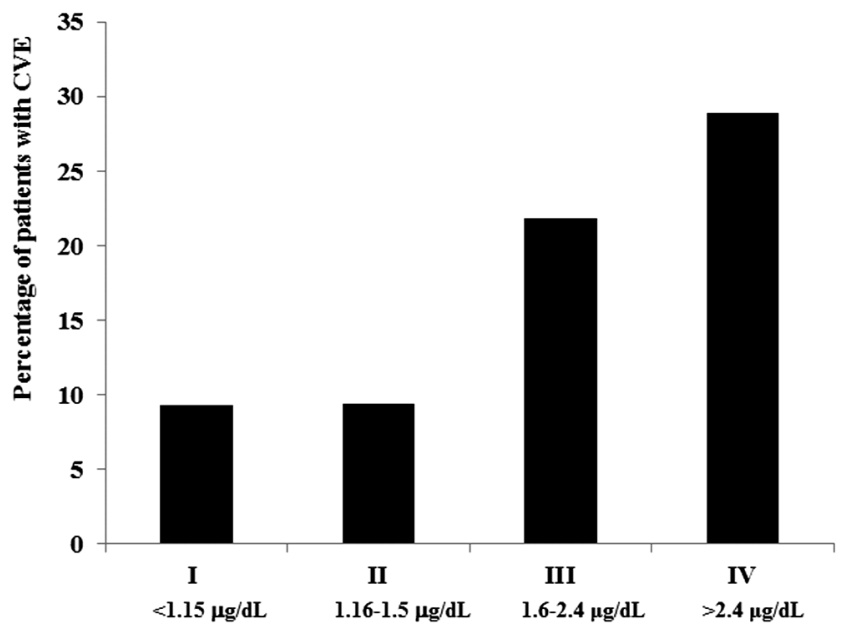

Quartiles of cortisol levels after 1mg-DST

\section{Figure 1}

Prevalence of cardiovascular events (CVE) among the whole sample of patients subdivided in quartiles on the basis of cortisol levels at 08:00 $\mathrm{h}$ after $1 \mathrm{mg}$-dexamethasone suppression test (1 mg-DST). In patients with cortisol after $1 \mathrm{mg}$-DST above $1.5 \mu \mathrm{g} / \mathrm{dL}$, the CVE prevalence progressively increased with increasing cortisol levels. The CVE prevalence was similar between I and II quartiles (9.3\% and 9.4\% respectively) and between the II and IV quartiles $(21.8 \%$ and $28.9 \%$ respectively) whereas it was significantly higher in the III and IV quartiles as compared with I and II quartiles, $P=0.01$ for both comparisons.

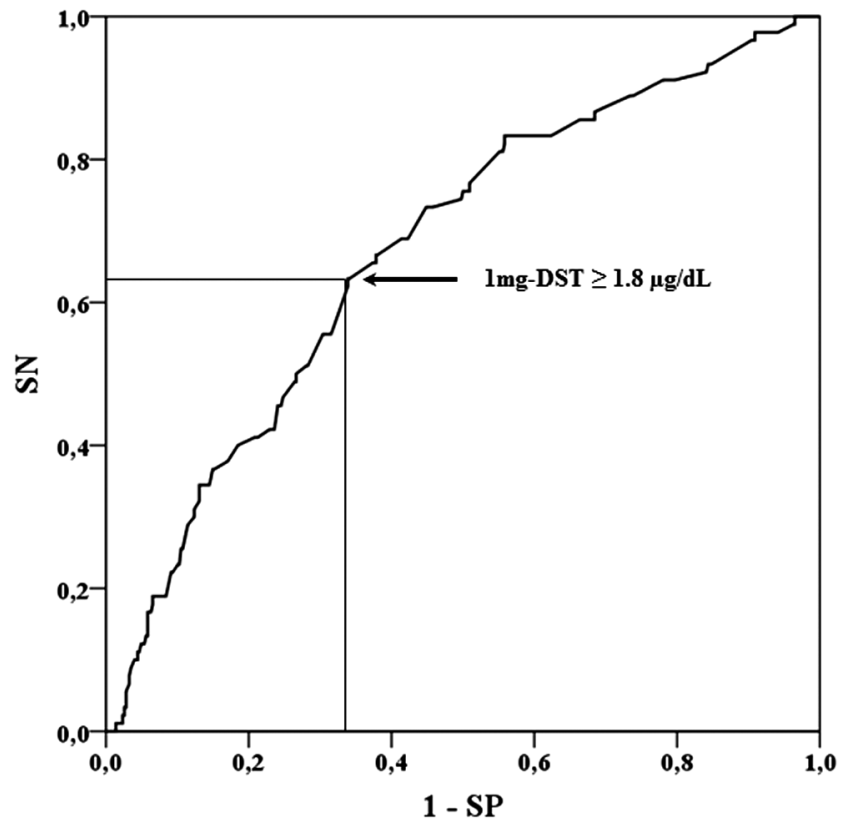

Figure 2

The receiver-operating characteristic (ROC) curve analysis showing the association between serum cortisol levels at 08:00 h after $1 \mathrm{mg}$-dexamethasone suppression test (1 mg-DST) and the occurrence of cardiovascular events. The ROC curve showed that the cut-off of cortisol levels after $1 \mathrm{mg}$-DST set at $1.8 \mu \mathrm{g} / \mathrm{dL}$ had the best compromise between sensitivity (SN, 63\%) and specificity (SP, 66\%) in predicting CVE (area under the curve, AUC, 0.673, $P<0.0001$ ).

The ROC curve analysis tested the optimal cut-off of the cortisol levels after $1 \mathrm{mg}$-DST for detecting patients with increased CVE risk and showed that the cut-off of cortisol levels after $1 \mathrm{mg}-\mathrm{DST}$ set at $1.8 \mu \mathrm{g} / \mathrm{dL}$ had the best compromise between SN (63\%) and SP (66\%) in predicting CVE (accuracy 62.9\%, area under the curve, AUC, 0.673, $P<0.0001$, Fig. 2). On the basis of this result, we, therefore, compared the clinical and biochemical characteristics of patients with cortisol levels after $1 \mathrm{mg}$-DST $\geq 1.8$ and $<1.8 \mu \mathrm{g} / \mathrm{dL}$ (DST,$+ n=220$ and DST-, $n=298$ respectively, Table 3). The two groups were comparable for gender distribution, BMI, familial CVE history and prevalence of $\mathrm{OB}$, smokers, postmenopausal women and of not well controlled blood pressure, glycemia and LDL. As expected, DST+ patients showed higher prevalence of h-UFC and lower ACTH levels than DST- patients. Age, adenoma size and $\mathrm{AH}, \mathrm{T} 2 \mathrm{DM}, \mathrm{DL}$ and CVE prevalence were higher in DST+ than in DST- patients. The CVE occurred within 2 years before or after the AI finding in $55.9 \%$ of DST+ patients and in $25.8 \%$ of DST- patients $(P=0.006)$. 
Table 3 Clinical and biochemical parameters of patients with and without $1 \mathrm{mg}-\mathrm{DST} \geq 1.8 \mu \mathrm{g} / \mathrm{dL}$. Data are mean $\pm \mathrm{s}$.d. with range in parenthesis or absolute number with percentage in parenthesis.

Age (years)
Male/female gender
Postmenopausal females
BMI (kg/m²)
Diameter of adenoma (mm)
Duration of observation period (months)
Patients with familiar history of CVE
Smokers
ACTH (pg/mL)
1 mg-DST ( $\mu \mathrm{g} / \mathrm{dL}$ )
h-UFC patients
Hypertensive patients
Blood pressure not well controlled
Diabetic patients
Glycemia not well controlled ${ }^{\mathrm{b}}$
Dyslipidemic patients
LDL not well controlled
Obese subjects
Patients with CVE

\begin{tabular}{c}
\hline DST $+(n=220) n=231$ \\
$66.0 \pm 10.2(20-85)$ \\
$84 / 136(38.2 / 61.8)$ \\
$125(56.8)$ \\
$27.4 \pm 4.9(18-40)$ \\
$28.8 \pm 9.5(10-60)$ \\
$163.7 \pm 44.9(120-353)$ \\
$84(28.2)$ \\
$96(43.6)$ \\
$12.3 \pm 8.5(1-55)$ \\
$3.1 \pm 1.6(1.8-11.5)$ \\
$27(12.3)$ \\
$164(74.5)$ \\
$96(43.6)$ \\
$57(25.9)$ \\
$15(6.8)$ \\
$98(41.4)$ \\
$54(25.4)$ \\
$62(28.2)$ \\
$59(26.8)$
\end{tabular}

\begin{tabular}{c}
\hline DST- $(n=298)$ \\
\hline $61.9 \pm 10.3(34-86)$ \\
$120 / 178(40.3 / 59.7)$ \\
$147(43.9)$ \\
$27.8 \pm 4.6(18.3-40)$ \\
$21.6 \pm 8.3(10-55)$ \\
$160.4 \pm 45.3(121-426)$ \\
$66(30)$ \\
$124(41.6)$ \\
$16.5 \pm 10.8(1.6-55)$ \\
$1.1 \pm 0.35(0.1-1.7)$ \\
$15(5.0)$ \\
$181(60.7)$ \\
$123(41.6)$ \\
$55(18.5)$ \\
$19(6.4)$ \\
$91(32.9)$ \\
$62(20.8)$ \\
$88(29.5)$ \\
$31(10.4)$ \\
\hline
\end{tabular}

\begin{tabular}{c}
\hline $\boldsymbol{P}$ \\
\hline$<0.001$ \\
0.650 \\
0.109 \\
0.30 \\
$<0.001$ \\
0.420 \\
0.695 \\
0.654 \\
$<0.001$ \\
$<0.001$ \\
0.003 \\
0.001 \\
0.653 \\
0.049 \\
0.859 \\
0.049 \\
0.338 \\
0.769 \\
$<0.001$ \\
\hline
\end{tabular}

${ }^{a}$ Among hypertensive patients; bamong diabetic patients.

$1 \mathrm{mg}$-DST, serum cortisol levels after $1 \mathrm{mg}$ dexamethasone suppression test; ACTH, adrenocorticotroph hormone; BMI, body mass index; CVE, cardiovascular event; DST+, patients with $1 \mathrm{mg}$-DST $\geq 1.8 \mu \mathrm{g} / \mathrm{dL}$; DST-, patients with $1 \mathrm{mg}$-DST $<1.8 \mu \mathrm{g} / \mathrm{dL}$; h-UFC, urinary free cortisol levels above normal reference range; LDL, low density lipoprotein; SI conversion factors, cortisol $\times 27.59$, ACTH $\times 0.22$; smokers, individuals who smoked any tobacco (including beedies, pipes and other forms) during the observational period.

In the whole sample, the comorbidities score was associated with the cortisol levels after $1 \mathrm{mg}$-DST $(r=0.105, P=0.017)$. Moreover, the CVE occurrence was directly associated with the increase of the comorbidity score $(0=2.8 \%, 1=13.6,2=21.2 \%, 3=28.9 \%, 4=44.4 \%$, $P$ for trend $<0.0001)$. The ROC curve analysis showed that a comorbidity score of 2 had the best compromise between SN (72.2\%) and SP (57.5\%) for detecting patients with increased CVE risk (AUC: $0.70, P<0.0001$ ) and that a comorbidity score $\geq 2$ had $60 \%$ accuracy for detecting patients with increased CVE risk. Adding the DST+ presence to the comorbidity score increased the accuracy to $71.2 \%$ (SN: 61.1, SP: 73.4\%). The logistic regression analysis showed that the CVE occurrence was 2.5 folds increased in DST+ patients, regardless of age, gender, duration of observation, familial CVE history, smoking habit, T2DM, AH and DL (Table 4, Panel A). This association was confirmed also when the comorbidity score $\geq 2$ was included into the regression analysis in the place of T2DM, AH and DL (Table 4, Panel B).

\section{Artificial neural networks analysis (ANNs)}

The connectivity map developed by Auto-CM system showed that the CVE occurrence was directly associated to the presence of cortisol levels after 1-DST $\geq 1.8 \mu \mathrm{g} / \mathrm{dL}$, $\mathrm{AH}$ and male gender (Fig. 3).
The following variables were put into the TWIST system: familial CVE history, age, gender, BMI, cortisol levels after $1 \mathrm{mg}$ DST, smoking packs/year, presence of postmenopausal status, smoking habit, obesity, hypertension, diabetes, well controlled blood pressure, glycemia and LDL, low ACTH levels (i.e. $<10 \mathrm{pg} / \mathrm{dL}$ ), h-UFC levels, $1 \mathrm{mg}$-DST $\geq 1.8 \mu \mathrm{g} / \mathrm{dL}$ and CVE occurrence. Among these variables, the TWIST system selected the CVE occurrence, familial CVE history, age, T2DM, AH, DL, low ACTH, h-UFC levels, $1 \mathrm{mg}$-DST $\geq 1.8 \mu \mathrm{g} / \mathrm{dL}$ and cortisol levels after $1 \mathrm{mg}$ DST. By using this cluster of variables supervised neural networks employing back propagation algorithm were able to detect patients with increased CVE risk with $78.5 \%$ accuracy (SN: 78.7\%, SP: 78.3\%). These performances are the average of two different training-testing experiments which allow all records to be classified in blind way.

By excluding $1 \mathrm{mg}$-DST $\geq 1.8 \mu \mathrm{g} / \mathrm{dL}$ the diagnostic accuracy decreased to $72.5 \%$ (SN: 70.9\%, SP: 74.1\%).

\section{Discussion}

The present study was designed to investigate if in patients with unilateral AI, the presence of an increased, though asymptomatic, cortisol secretion was independently associated with the CVE occurrence and if ANNs could be useful for detecting patients with increased CVE risk. 
Table 4 Association between serum cortisol levels after $1 \mathrm{mg}$ dexamethasone suppression test $\geq 1.8 \mu \mathrm{g} / \mathrm{dL}$ and the occurrence of cardiovascular event after adjustment for the presence of comorbidities (Model A) or of the comorbidities score (Model B) and other possible contributing factors using the multivariate logistic regression analysis.

\begin{tabular}{|c|c|c|c|}
\hline & Odds ratio & $95 \% \mathrm{Cl}$ & $P$ value \\
\hline \multicolumn{4}{|l|}{ Model A } \\
\hline Female gender & 1.6 & $0.9-2.7$ & 0.13 \\
\hline Age (1 year increase) & 1.03 & $1.0-1.1$ & 0.023 \\
\hline $\begin{array}{l}\text { Familiar history of CVE } \\
\text { (presence vs absence) }\end{array}$ & 1.1 & $0.6-1.9$ & 0.724 \\
\hline T2DM (presence vs absence) & 1.9 & $1.1-3.4$ & 0.025 \\
\hline $\mathrm{AH}$ (presence vs absence) & 4.3 & $1.8-9.8$ & 0.001 \\
\hline DL (presence vs absence) & 1.8 & $1.1-3.1$ & 0.032 \\
\hline Smoke (presence vs absence) & 1.8 & $1.1-3.2$ & 0.03 \\
\hline $\begin{array}{l}\text { Duration of observation } \\
\text { period ( } 1 \text { month increase) }\end{array}$ & 1.0 & $1.0-1.0$ & 0.489 \\
\hline $\begin{array}{l}1 \mathrm{mg}-\mathrm{DST} \geq 1.8 \mu \mathrm{g} / \mathrm{dL} \\
\text { (presence vs absence) }\end{array}$ & 2.5 & $1.5-4.2$ & $<0.001$ \\
\hline \multicolumn{4}{|l|}{ Model B } \\
\hline Female gender & 1.7 & $1.0-2.9$ & 0.04 \\
\hline Age (1 year increase) & 1.0 & $1.0-1.1$ & 0.001 \\
\hline $\begin{array}{l}\text { Familiar history of CVE } \\
\text { (presence vs absence) }\end{array}$ & 1.1 & $0.6-1.9$ & 0.740 \\
\hline Comorbidity score $(<2$ or $\geq 2)$ & 2.9 & $1.7-4.9$ & $<0.001$ \\
\hline Smoke (presence vs absence) & 1.6 & $1.0-2.9$ & 0.053 \\
\hline $\begin{array}{l}\text { Duration of observation } \\
\text { period ( } 1 \text { month increase) }\end{array}$ & 1.0 & $1.0-1.0$ & 0.638 \\
\hline $\begin{array}{l}1 \mathrm{mg}-\mathrm{DST} \geq 1.8 \mu \mathrm{g} / \mathrm{dL} \\
\text { (presence vs absence) }\end{array}$ & 2.7 & $1.6-4.5$ & $<0.001$ \\
\hline
\end{tabular}

$1 \mathrm{mg}$-DST, serum cortisol levels at 08:00 $\mathrm{h}$ after $1 \mathrm{mg}$ dexamethasone suppression test; $\mathrm{AH}$, arterial hypertension; comorbidities score, sum of the comorbidities (type 2 diabetes, arterial hypertension, dyslipidemia; $\mathrm{DL}$, dyslipidemia; smoke, smoke of any tobacco (including beedies, pipes and other forms) during the observational period; T2DM, type 2 diabetes mellitus.

We found that the CVE prevalence is 1.3 -fold increased for each $1 \mathrm{mg}$ increase of cortisol after $1 \mathrm{mg}$-DST, regardless for the known cardiovascular risk factors. Moreover, the cut-off of cortisol levels after $1 \mathrm{mg}$-DST set at $1.8 \mu \mathrm{g} /$ $\mathrm{dL}$, as established by ROC curve, had the best accuracy for detecting patients with increased CVE risk and that it confers a 2.5-folds increased risk of CVE. The ANNs confirmed the independent association between DST+ and the CVE risk and, as compared with the standard statistics, they show, at the same SP (73.4 and 78.7\% respectively), higher $\mathrm{SN}$ (78.7\% and $61.1 \%$ respectively) for detecting patients with increased CVE risk.

The present findings are in keeping with those of a previous smaller study by Di Dalmazi and co-authors (9) and with those of a previous Italian multicenter study (10) showing that AI patients with mild ACS (possible or ascertained) had an increased CVE occurrence. Although in those studies the association between mild ACS and the CVE was present regardless for hypertension (9) and age $(9,10)$, the additive role of mild ACS on the CVE occurrence was not completely clarified. Indeed, the difficulties in determining the influence of mild ACS on the cardiovascular risk have been also due to the lack of a widely accepted definition of mild ACS itself $(17,37)$. In particular, in the previous studies the mild ACS condition was diagnosed on the basis of arbitrarily established cut-offs of the cortisol levels after $1 \mathrm{mg}$-DST $(8,10)$ or combination of parameters of hypothalamic-pituitaryadrenal axis activity (8). At variance, in the present study, thanks to its large sample of patients, the patients were not arbitrarily classified as affected or not with mild ACS, whereas we let the ROC curve independently identify the cut-off of cortisol levels after $1 \mathrm{mg}$-DST with the best accuracy for detecting patients with increased CVE risk. By using this different, and, in our opinion, less biased approach, we were able to confirm that the cut-off of cortisol levels after $1 \mathrm{mg}$-DST above or equal to $1.8 \mu \mathrm{g} /$ $\mathrm{dL}$ was the most accurate one for detecting patients with increased CVE risk. Therefore, it could be hypothesized that this cut-off of cortisol levels after $1 \mathrm{mg}$-DST could be considered for diagnosing mild ACS. However, the present data also show that in AI patients with cortisol levels after $1 \mathrm{mg}$-DST above $1.5 \mu \mathrm{g} / \mathrm{dL}$ the CVE prevalence progressively increases with increasing cortisol levels after $1 \mathrm{mg}$-DST, but also that it might be particularly high in patients with cortisol levels after $1 \mathrm{mg}$ DST above $3.1 \mu \mathrm{g} / \mathrm{dL}$.

Apart from the mild ACS definition, the main problem with the use of the biochemical tests in AI subjects is that raising the cut-offs of the cortisol secretion markers increases SP, but at the expense of a not acceptable SN decrease and vice versa. Interestingly, a recent metaanalysis suggests a beneficial effect of adrenalectomy on cardiovascular risk factors in patients with mild ACS as compared with the conservative management, even if with a low-moderate quality of evidence (38). However, to date, we cannot use a combination of clinical and biochemical markers for predicting the cardiovascular outcome in these patients; therefore, an algorithm for assessing the cardiovascular risk in the individual AI patient is lacking (16). These difficulties are mainly due to the fact that some comorbidities (i.e. T2DM, AH, DL and $\mathrm{OB})$, which may depend even on mild ACS itself, may worsen the mild ACS-related cardiovascular risk and interact with mild ACS in nonlinear biologic mechanisms.

From this point of view, the evaluation of the possible use of the ANNs to investigate the relationships between mild ACS and the common cardiovascular risk factors is a 


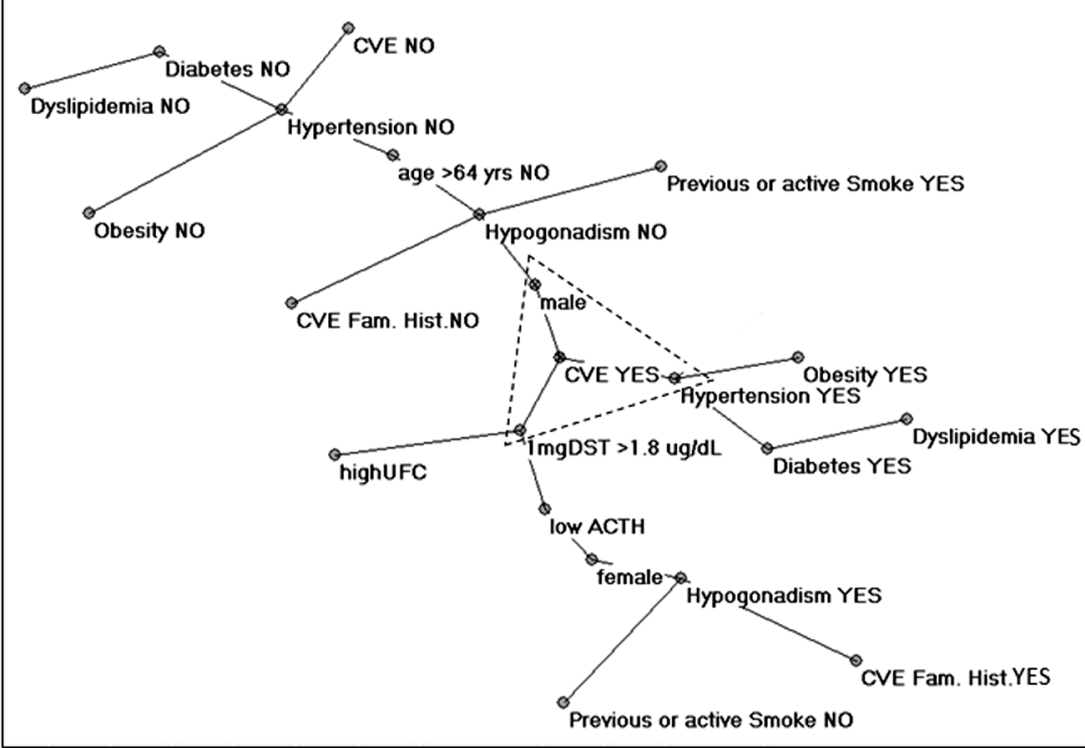

\section{Figure 3}

Semantic connectivity map of studied variables. The semantic connectivity map showed that the occurrence of a cardiovascular event (CVE) was directly associated to the presence of cortisol levels at 08:00 $\mathrm{h}$ after $1 \mathrm{mg}$-dexamethasone suppression test ( $1 \mathrm{mg}$-DST) $\geq 1.8 \mu \mathrm{g} / \mathrm{dL}$, arterial hypertension and male gender (included within a triangle). novel approach. The ANNs are artificial adaptive systems, able to modify their internal structure in relation to a function objective, and, therefore, they are particularly suited for solving nonlinear problems (20). The ANNs analysis suggests that combining several variables, including the cardiovascular risk factors, age and cortisol levels after $1 \mathrm{mg}$-DST, it might be possible to increase the sensitivity (up to about 79\%) for detecting patients with increased CVE risk. However, the actual advantage of the ANNs should be demonstrated by comparing their accuracy with that of the available cardiovascular risk calculators, which could not have been applied in the present study. To date, the ANNs approach is hardly applicable in the clinical practice due to its not entirely satisfactory diagnostic overall accuracy. However, the ANNs could open the field for the development of algorithms for assessing the CVE risk in the individual AI patient, by prospectively studying much larger sample of patients. Indeed, a reliable assessment of the CVE risk in the individual AI patient could consent to personalize the treatment of the comorbidities and, thus, to reduce the CVE risk itself.

Nevertheless, the present results still have an important clinical implication. Indeed, they suggest that in AI patients, in the presence of comorbidities potentially related to hypercortisolism, the finding of cortisol levels after $1 \mathrm{mg}$-DST above $1.8 \mu \mathrm{g} / \mathrm{dL}$ could indicate the need of a strict monitoring of the cardiovascular risk. However, given the suboptimal accuracy of both the classical statistics and ANNs approach for detecting patients with increased CVE risk, it remains difficult to estimate the individual CVE risk in the single AI patient.

This study has some limitations. Firstly, we acknowledge that our study is cross-sectional and it does not report data regarding the CVE incidence during a standardized follow-up. In addition, being the mild ACS condition asymptomatic, we cannot have information about the temporal distance between the onset of hypercortisolism and the CVE occurrence. However, at variance with DST- patients, in DST+ patients the CVE occurred mainly within 2 years before or after the AI finding. This finding suggests a temporal relationship between the CVE occurrence and the condition of subtle hypercortisolism and confirms the results of a previous smaller study showing that mild ACS is associated with the risk of incident CVE (9). In contrast, given that more than 50\% of CVE occur within 2 years from the $\mathrm{SH}$ diagnosis it remains unclear if the CVE may be prevented by curing mild ACS.

Furthermore, we have no information regarding the behavior of the cortisol secretion and the comorbidities of the subjects during the observation period, and, therefore, we could not assess the influence of the possible increase of the cortisol secretion and/or of the possible changes in the comorbidities control. However, since the comorbidities control assessed at the study entry was not different between patients with and without CVE and with and without DST+, this variable should not have been of particularly influence in the study. Similarly, even if the older age of DST+ patients as compared with DST- ones 
could have played a role in increasing the CVE risk in the former group, the logistic regression analysis showed that the CVE risk remained associated with $1 \mathrm{mg}$-DST even after adjusting for age. Finally, given the cross-sectional design of the study, definitive information regarding the possible causative role of a subtle increased cortisol secretion on the occurrence of CVE could not be derived. However, in consideration of the known deleterious effect of the overt hypercortisolism on the CVE risk, a similar role of mild ACS could be affirmed.

In conclusion, the present study shows that: (i) a subtle cortisol excess is associated with the CVE occurrence regardless of age, gender, familial CVE history, AH, T2D, $\mathrm{DL}$ and smoke, therefore representing an additional CVE risk factor; (ii) the CVE prevalence is 1.3-fold increased for each $1 \mathrm{mg}$ increase of cortisol after $1 \mathrm{mg}$-DST, regardless for the known cardiovascular risk factors; (iii) the cut-off of cortisol levels after $1 \mathrm{mg}$-DST above or equal to $1.8 \mu \mathrm{g} /$ dL has the best accuracy in detecting AI patients with CVE; (iv) the application of the ANNs in evaluating the interaction between the cortisol secretion and the other CVE risk factor might increase the sensitivity for detecting patients with increased CVE risk.

\section{Declaration of interest}

The authors declare that there is no conflict of interest that could be perceived as prejudicing the impartiality of this study.

\section{Funding}

This research did not receive any specific grant from any funding agency in the public, commercial or not-for-profit sector. No author has been paid by pharmaceutical companies or other agencies.

\section{References}

1 Fassnacht M, Arlt W, Bancos I, Dralle H, Newell-Price J, Sahdev A, Tabarin A, Terzolo M, Tsagarakis S \& Dekkers OM. Management of adrenal incidentalomas: European Society of Endocrinology Clinical Practice Guideline in collaboration with the European Network for the Study of Adrenal Tumors. European Journal of Endocrinology 2016 175 G1-G34. (doi:10.1530/EJE-16-0467)

2 Chiodini I. Clinical review: diagnosis and treatment of subclinical hypercortisolism. Journal of Clinical Endocrinology and Metabolism 2011 96 1223-1236. (doi:10.1210/jc.2010-2722)

3 Debono M \& Newell-Price J. Subclinical hypercortisolism in adrenal incidentaloma. Current Opinion in Endocrinology Diabetes and Obesity 201522 185-192. (doi:10.1097/MED.0000000000000151)

4 Goddard GM, Ravikumar A \& Levine AC. Adrenal mild hypercortisolism. Endocrinology Metabolism Clinics of North America 201544 371-379. (doi:10.1016/j.ecl.2015.02.009)

5 Chiodini I, Vainicher CE, Morelli V, Palmieri S, Cairoli E, Salcuni AS, Copetti M \& Scillitani A. Endogenous subclinical hypercortisolism and bone: a clinical review. European Journal of Endocrinology $2016 \mathbf{1 7 5}$ R265-R282. (doi:10.1530/EJE-16-0289)
6 Giordano R, Guaraldi F, Berardelli R, Karamouzis I, D’Angelo V, Marinazzo E, Picu A, Ghigo \& Arvat E. Glucose metabolism in patients with subclinical Cushing's syndrome. Endocrine 201241 415-423. (doi:10.1007/s12020-012-9628-9)

7 Di Dalmazi G \& Pasquali R. Adrenal adenomas, subclinical hypercortisolism, and cardiovascular outcomes. Current Opinion in Endocrinology Diabetes and Obesity 201522 163-168. (doi:10.1097/ MED.0000000000000153)

8 Di Dalmazi G, Vicennati V, Rinaldi E, Morselli-Labate AM, Giampalma E, Mosconi C, Pagotto U \& Pasquali R. Progressively increased patterns of subclinical cortisol hypersecretion in adrenal incidentalomas differently predict major metabolic and cardiovascular outcomes: a large cross-sectional study. European Journal of Endocrinology 2012166 669-677. (doi:10.1530/EJE-11-1039)

9 Morelli V, Reimondo G, Giordano R, Della Casa S, Policola C, Palmieri S, Salcuni AS, Dolci A, Mendola M, Arosio M et al. Long-term follow-up in adrenal incidentalomas: an Italian multicenter study. Journal of Clinical Endocrinology and Metabolism 201499 827-834. (doi:10.1210/jc.2013-3527)

10 Di Dalmazi G, Vicennati V, Garelli S, Casadio E, Rinaldi E, Giampalma E, Mosconi C, Golfieri R, Paccapelo A, Pagotto U et al. Cardiovascular events and mortality in patients with adrenal incidentalomas that are either non-secreting or associated with intermediate phenotype or subclinical Cushing's syndrome: a 15-year retrospective study. Lancet Diabetes Endocrinology 20142 396-405. (doi:10.1016/S22138587(13)70211-0)

11 Debono M, Bradburn M, Bull M, Harrison B, Ross RJ \& NewellPrice J. Cortisol as a marker for increased mortality in patients with incidental adrenocortical adenomas. Journal of Clinical Endocrinology and Metabolism 201499 4462-4467. (doi:10.1210/jc.2014-3007)

12 Toniato A, Merante-Boschin I, Opocher G, Pelizzo MR, Schiavi F \& Ballotta E. Surgical versus conservative management for subclinical Cushing syndrome in adrenal incidentalomas: a prospective randomized study. Annals of Surgery 2009249 388-391. (doi:10.1097/ SLA.0b013e31819a47d2)

13 Chiodini I, Morelli V, Salcuni AS, Eller-Vainicher C, Torlontano M, Coletti F, Iorio L, Cuttitta A, Ambrosio A, Vicentini L et al. Beneficial metabolic effects of prompt surgical treatment in patients with an adrenal incidentaloma causing biochemical hypercortisolism. Journal of Clinical Endocrinology and Metabolism 201095 2736-2745. (doi:10.1210/jc.2009-2387)

14 Sereg M, Szappanos A, Toke J, Karlinger K, Feldman K, Kaszper E, Varga I, Gláz E, Rácz K \& Tóth M. Atherosclerotic risk factors and complications in patients with non-functioning adrenal adenomas treated with or without adrenalectomy: a long-term follow-up study. European Journal of Endocrinology 2009160 647-655. (doi:10.1530/EJE08-0707)

15 Morelli V, Scillitani A, Arosio M \& Chiodini I. Follow-up of patients with adrenal incidentaloma, in accordance with the European society of endocrinology guidelines: could we be safe? Journal of Endocrinological Investigation 201740 331-333. (doi:10.1007/ s40618-016-0558-x)

16 Chiodini I, Albani A, Ambrogio AG, Campo M, De Martino MC, Marcelli G, Morelli V, Zampetti B, Colao A, Pivonello R et al. Six controversial issues on subclinical Cushing's syndrome. Endocrine 201756 262-266. (doi:10.1007/s12020-016-1017-3)

17 Shen J, Sun M, Zhou B \& Yan J. Nonconformity in the clinical practice guidelines for subclinical Cushing's syndrome: which guidelines are trustworthy? European Journal of Endocrinology 2014171 421-431. (doi:10.1530/EJE-14-0345)

18 Eller-Vainicher C, Morelli V, Salcuni AS, Battista C, Torlontano M, Coletti F, Iorio L, Cairoli E, Beck-Peccoz P, Arosio M et al. Accuracy of several parameters of hypothalamic-pituitary-adrenal axis activity in predicting before surgery the metabolic effects of the removal of an adrenal incidentaloma. European Journal of Endocrinology 2010163 925-935. (doi:10.1530/EJE-10-0602) 
19 Di Dalmazi G, Pasquali R, Beuschlein F \& Reincke M. Subclinical hypercortisolism: a state, a syndrome, or a disease? European Journal of Endocrinology 2015173 M61-M71. (doi:10.1530/EJE-15-0272)

20 Buscema M \& Grossi E. The semantic connectivity map: an adapting self-organising knowledge discovery method in data bases. Experience in gastro-oesophageal reflux disease. International Journal of Data Mining and Bioinformatics 20082 362-404. (doi:10.1504/ IJDMB.2008.022159)

21 Buscema M, Grossi E, Snowdon D \& Antuono P. Auto-Contractive Maps: an artificial adaptive system for data mining. An application to Alzheimer disease. Current Alzheimer Research 20085 481-498. (doi:10. 2174/156720508785908928)

22 Buscema MBM \& Sacco PL. Auto-Contractive Maps, the H Function, and the Maximally Regular Graph (MRG): A New Methodology for Data Mining Applications of Mathematics in Models, Artificial Neural Networks and Arts, pp 227-275. Dordrecht: Springer, 2010. (doi:10.1007/978-90-481-8581-8_11)

23 Buscema M, Breda M \& Lodwick W. Training with Input Selection and Testing (TWIST) algorithm: a significant advance in pattern recognition performance of machine learning. Journal of Intelligent Learning Systems and Applications 20135 29-38. (doi:10.4236/ jilsa.2013.51004)

24 Buscema M, Grossi E, Capriotti M, Babiloni C \& Rossini PM. The I.F.A.S.T. model allows the prediction of conversion to Alzheimer disease in patients with mild cognitive impairment with high degree of accuracy. Current Alzheimer Research 20107 173-187. (doi:10.2174/ 156720510790691137)

25 Rotondano G, Cipolletta L, Grossi E, Koch M, Intraligi M, Buscema M, Marmo R, Italian Registry on Upper Gastrointestinal Bleeding. Artificial neural networks accurately predict mortality in patients with non variceal upper GI bleeding. Gastrointestinal Endoscopy 201173 218-226. (doi:10.1016/j.gie.2010.10.006)

26 Obermeyer Z \& Emanuel EJ. Predicting the future-big data, machine learning, and clinical medicine. New England Journal of Medicine 2016 375 1216-1219. (doi:10.1056/NEJMp1606181)

27 Eller-Vainicher C, Chiodini I, Santi I, Massarotti M, Pietrogrande L, Cairoli E, Beck-Peccoz P, Longhi M, Galmarini V, Gandolini G et al. Recognition of morphometric vertebral fractures by artificial neural networks: analysis from GISMO Lombardia Database. PLOS ONE 2011 6 e27277. (doi:10.1371/journal.pone.0027277)

28 Eller-Vainicher C, Zhukouskaya VV, Tolkachev YV, Koritko SS, Cairoli E, Grossi E, Beck-Peccoz P, Chiodini I \& Shepelkevich AP. Low bone mineral density and its predictors in type 1 diabetic patients evaluated by the classic statistics and artificial neural network analysis. Diabetes Care 201134 2186-2191. (doi:10.2337/dc11-0764)

29 Baldassarre D, Grossi E, Buscema M, Intraligi M, Amato M, Tremoli E, Pustina L, Castelnuovo S, Sanvito S, Gerosa
L et al. Recognition of patients with cardiovascular disease by artificial neural networks. Annals of Medicine 200436 630-640. (doi:10.1080/07853890410018880)

30 Kreitschmann-Andermahr I, Psaras T, Tsiogka M, Starz D, Kleist B, Siegel S, Milian M, Kohlmann J, Menzel C, Führer-Sakel D et al. From first symptoms to final diagnosis of Cushing's disease: experiences of 176 patients. European Journal of Endocrinology 2015172 285-289. (doi:10.1530/EJE-14-0766)

31 The Expert Committee on the Diagnosis and Classification of Diabetes Mellitus. Report of the expert committee on the diagnosis and classification of diabetes mellitus. Diabetes Care 200326 (Supplement 1) S5-S20. (doi:10.2337/diacare.26.2007.s5)

32 Task Force on diabetes, pre-diabetes, and cardiovascular diseases of the European Society of Cardiology (ESC), European Association for the Study of Diabetes (EASD), Rydén L, Grant PJ, Anker SD, Berne C, Cosentino F, Danchin N, Deaton C, Escaned J et al. ESC guidelines on diabetes, pre-diabetes, and cardiovascular diseases developed in collaboration with the EASD - summary. Diabetes and Vascular Disease Research 201411 133-173. (doi:10.1177/1479164114525548)

33 Mancia G, Fagard R, Narkiewicz K, Redon J, Zanchetti A, Böhm M, Christiaens T, Cifkova R, De Backer G, Dominiczak A et al. 2013 ESH/ESC Practice Guidelines for the management of arterial hypertension. Blood Pressure 201423 3-16. (doi:10.3109/08037051.2 014.868629)

34 Expert Panel on Detection, Evaluation, and Treatment of High Blood Cholesterol in Adults. Executive summary of the third report of the national cholesterol education program (NCEP) expert panel on detection, evaluation and treatment of high blood cholesterol in adults (adult treatment panel III). JAMA 2001285 2486-2497. (doi:10.1001/jama.285.19.2486)

35 Teo KK, Ounpuu S, Hawken S, Pandey MR, Valentin V, Hunt D, Diaz R, Rashed W, Freeman R, Jiang L et al. Tobacco use and risk of myocardial infarction in 52 countries in the INTERHEART study: a case-control study. Lancet 200619368 647-658. (doi:10.1016/S01406736(06)69249-0)

36 Box GEP \& Tidwell PW. Transformation of the independent variables. Technometrics 19624 531-550. (doi:10.1080/00401706. 1962.10490038)

37 Morelli V \& Chiodini I. Subclinical hypercortisolism: how to deal with it. Frontiers of Hormone Research 201646 28-38. (doi:10.1159/000443862)

38 Bancos I, Alahdab F, Crowley RK, Chortis V, Delivanis DA, Erickson D, Natt N, Terzolo M, Arlt W, Young WF et al. Improvement of cardiovascular risk factors after adrenalectomy in patients with adrenal tumors and subclinical Cushing's syndrome: a systematic review and meta-analysis. European Journal of Endocrinology 2016175 R283-R295. (doi:10.1530/EJE-16-0465)

Received 20 January 2017

Revised version received 18 April 2017

Accepted 2 May 2017 